\title{
TESTING THE SUSCEPTIBILITY OF ROLLING BEARING VIBRATION MEASUREMENT SYSTEMS
}

\author{
Mateusz Wrzochal, Stanisław Adamczak, Henryk Łomża, Ryszard Łuszczak
}
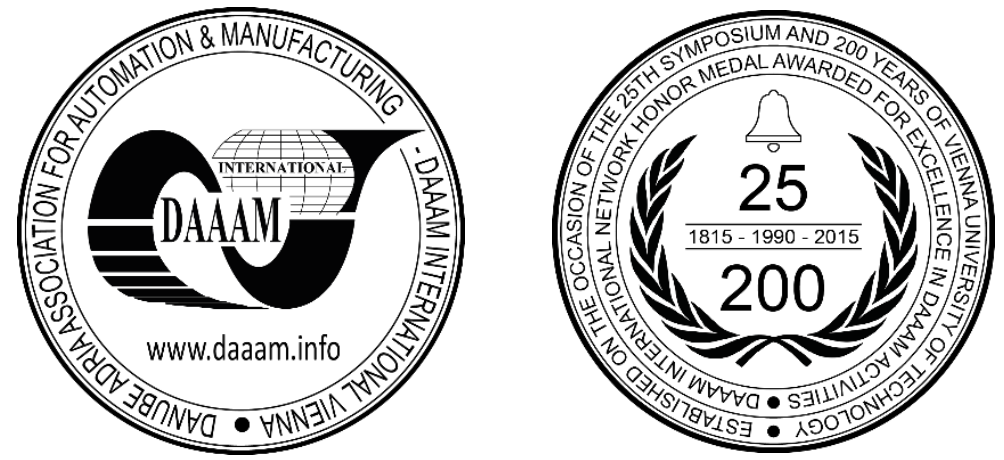

This Publication has to be referred as: Wrzochal, M[ateusz]; Adamczak, S[tanislaw]; Lomza, H[enryk] \& Luszczak, R[yszard] (2018). Testing the Susceptibility of Rolling Bearing Vibration Measurement Systems, Proceedings of the 29th DAAAM International Symposium, pp.0860-0865, B. Katalinic (Ed.), Published by DAAAM International, ISBN 978-3-902734-20-4, ISSN 1726-9679, Vienna, Austria

DOI: $10.2507 / 29$ th.daaam.proceedings.124

\begin{abstract}
Leading manufacturers of rolling bearings have their own vibration measurement systems that involve several key components. Although the method of operation is the same for all the systems, they differ in terms of some design solutions and the type of design affects the accuracy of the measurements of vibrations in a rolling bearing. The paper continues the discussion on the issues related to rolling bearing vibration measurements, focusing on the susceptibility of rolling bearing vibration measurement systems to the level of vibration. The analysis of sources of measurement errors is vital in studies focusing on the evaluation of metrological properties of industrial-grade systems for measuring the bearing vibration level.
\end{abstract}

Keywords: rolling bearings; vibrations; measurements; systems accuracy

\section{Introduction}

Available publications often deal with the diagnosing the condition of rolling bearings based on the measurement of generated vibrations [1], [2], [3], [4]. Equally often, we can find articles focusing on tests of vibration sensors, used to measure bearing vibrations [5], [6], [7]. Complete industrial measurement systems are a rare subject of open research, and problems related to them are solved inside the factories. And yet the decision whether the bearing will be allowed to work, is taken in industrial plants.

The measurement of rolling bearing vibration is one of the most important parts of quality control in bearing production facilities [8], [9]. Global manufacturers of rolling bearings develop their own control stations or have their own suppliers of special-purpose measurement instruments. In short, the industrial-level measurement of rolling bearing vibration involves recording the velocity of radial vibration of a bearing on a shaft that rotates at a specific speed. To this end, electrodynamic sensors are used, which generate signals proportional to the vibration velocity. During the measurement, a rolling bearing is axially preloaded with a force appropriate to the type and size of the bearing. The quality of the bearing is assessed based on the calculated RMS value of the signal in three filtered frequency bands: low, medium, and high. Each band can help detect different potential defects characteristic of rolling bearings. 
Abnormally large vibration level in the low band, for example, may be indicative of such issues as the difference in ball diameter or ring roundness; the medium band can help detect a ball flaw or race waviness; and the high band can be used to detect excessive ball or race roughness or contaminations. [10], [11], [12].

The level of vibration produced by a bearing should meet customer expectations. Standards for measuring rolling bearing vibration level specify only the general conditions for measurements such as the rotational speed of the inner ring of the bearing, the force applied to the outer ring, or the filtering of the resulting signal [13], [14]. The standards fail to determine the technical means to ensure appropriate measurement conditions. Therefore, the existing systems differ in terms of some design solutions of their components.

There are various types of designs of the components in question. An analysis of just some of the available devices [10], [11], [15], [16], [17], [18] indicates that the differences may involve: the type of spindle, type of preloading device, type of preload-outer ring contact, the location of the motor, sensor mounting, or the manner of processing of the measurement signal. Each of the above-mentioned components affects measurement accuracy to some extent. A diagram of factors affecting the accuracy of a bearing vibration measurement system is shown in Fig. 1 .

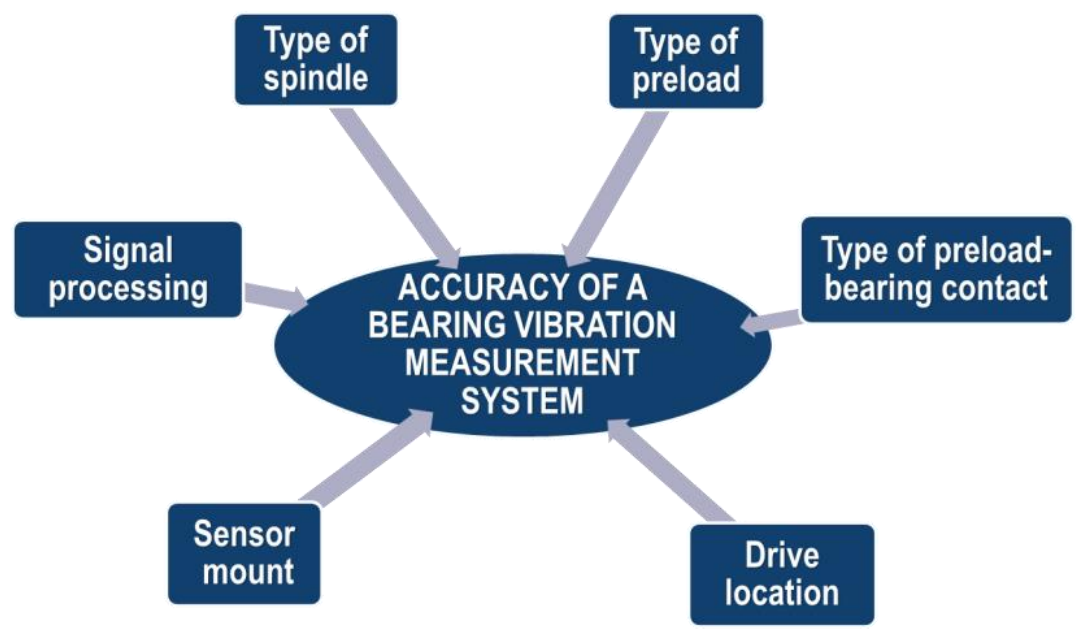

Fig. 1. Factors affecting the accuracy of a bearing vibration measurement system

The goal of the study was to verify whether a specific design of a measurement system can be susceptible to the level of bearing vibration. It is, of course, related to the fact whether the measured bearing was damaged during production or is free of major flaws. The study compared three industrial-grade systems for measuring rolling bearing vibration. Their specifications are listed below:

- Tested measurement system 1: This system has a hydrodynamic spindle as well. The preload is applied by a pneumatic actuator fixed to a compact structure to ensure a constant coaxiality of the preload and the spindle. The preload head touches the outer ring of the tested bearing over its whole perimeter. Any misalignment is compensated for by a rubber element on the perimeter of the preload ring in contact with the bearing. The drive is located far from the spindle and the revolving movement is transmitted via a V-belt. The sensor is fixed to a movable system with a small pneumatic actuator. This way, the sensor is in contact with the bearing only during the measurement. When no measurement is being performed, the actuator pulls the sensor away, thus preventing unnecessary wear and tear or accidental damage. The system offers signal visualisation and logging of measurement results in a digital format.

- Tested measurement system 2: The spindle in the system has hydrodynamic bearings. The preload is applied and released by a pneumatic actuator on a movable arm. As a result, the preload and the spindle are coaxial only during the measurement. The preload head contacts the bearing at three points. Any misalignment is compensated for by a joint between the pressure plate and the actuator. The drive is located far from the spindle and the revolving movement is transmitted via a V-belt. The electrodynamic sensor is fixed in a mount over the spindle and bearings slide on its mandrel. The system has an analogue-to-digital converter for logging measurement results in a digital format.

- Reference measurement system [19]: The spindle of the reference measurement system is supported completely by air bearings. The preload is applied and released with a pneumatic actuator in a compact structure ensuring a constant coaxiality of the preload and the spindle. The preload head contacts the bearing at three points. Any misalignments are compensated for by a joint between the pressure plate and the actuator. The drive is directly behind the spindle and transmits the revolutions through a clutch. The transducer is fixed in a mount over the spindle and tested bearings slide on the mandrel of the sensor. The measurement signal in the reference system undergoes analogue processing. Measurement results can be read only from gauges on a panel after correct settings are selected with knobs. This solution requires substantial expertise on the part of the operator when reading results. 


\section{Methods of measurements}

The study involved two bearings $6204 \mathrm{ZZ}$ and 6205ZZ. The first one generated a relatively low level of vibration and noise assessment did not identify substantial damage. Bearing 6205ZZ generated an excessive vibration level. The noise assessment for this bearing identified inaccuracy in outer ring race structure. The rotational speed was each time 1,800 RPM and preload was $60 \mathrm{~N}$ as per accepted standards. Pneumatic preload was released and the reapplied between measurements.

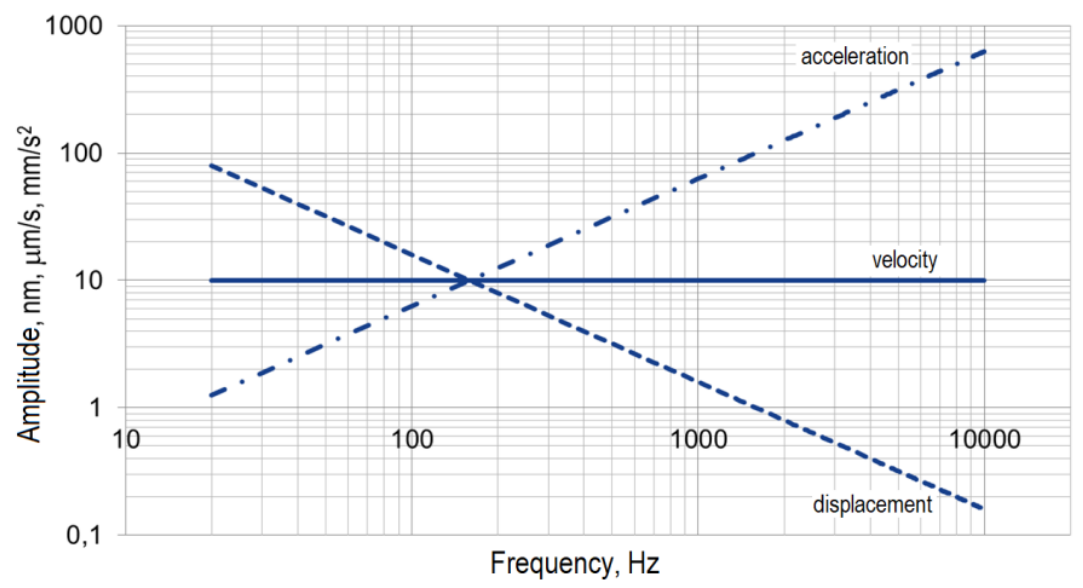

Fig. 2. Amplitude relation between displacement, velocity, and acceleration

The measurement signal is normally filtered into three frequency bands: low 50-300 Hz, medium 300-1,800 Hz, and high 1,800-10,000 Hz. The RMS value of vibration is calculated for each band. In this analysis, only the medium band vibrations are assessed. This simplification was introduced because all the test stands employ electrodynamic vibration sensors that register vibration velocity directly. When analysing the amplitude relationships between displacement, velocity, and acceleration for sine movement (Fig. 2), it is clear that in the low-frequency band, it is the displacement that offers the best detectable amplitude, while in the very high-frequency band, it is acceleration. Vibration velocity is best measured in the medium band. The RMS value of vibration in the medium band is expressed in a special unit, Anderon, which is used mainly in the bearing industry. This value is closely related to the rotational speed. The relationship between the initial vibration velocity unit ( $\mu \mathrm{m} / \mathrm{s})$ and Anderon (And) [9],[19],[20] is expressed by (1):

$$
1 \text { And }=2 \pi \cdot 30 \sqrt{\log _{2} \frac{f_{h}}{f_{l}}} \mu \mathrm{m} / \mathrm{s}
$$

where:

$f_{h}$ - the upper limit of the frequency band, $\mathrm{Hz}$;

$f_{l}$ - the lower limit of the frequency band, Hz.

In order to determine the nature of the dependence between results for the investigated systems, 20 measuring points were marked on the outer ring of the tested bearing at fixed intervals (Fig. 3).

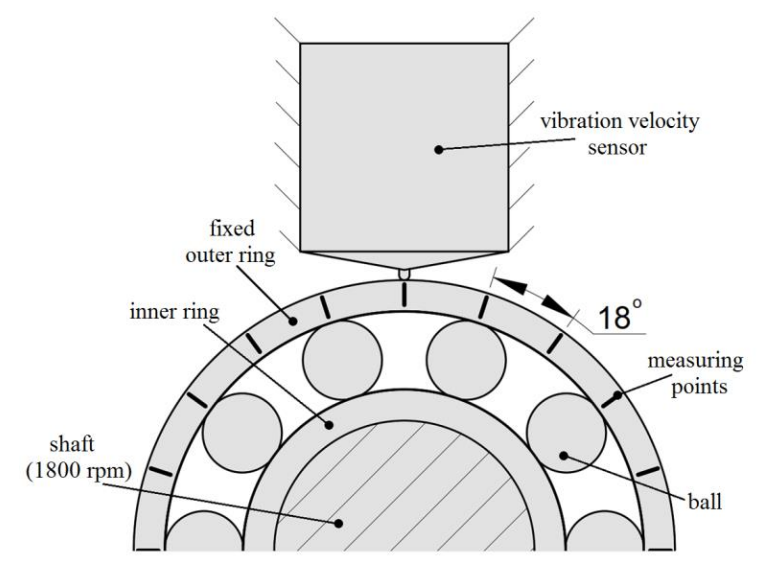

Fig. 3. Diagram of bearing vibration measurement at multiple points on the perimeter of the outer ring 
The vibration level at a given point on the bearing depends mainly on the geometrical structure of the race in the outer ring. This is because the sensor contact element is fixed in one place. Other components of the rolling bearing (the inner ring, cage, and balls) revolve in cycles. If the operation of the systems does not affect the results, one could expect at least a correlation between individual points.

\section{Results}

Results of the measurements of the RMS value of vibrations at points on the perimeter of the outer ring are shown in the charts in Fig. 4. For measurements of bearing 6204ZZ, there is a convergence of system 1 and reference system results. It may be that the variability of the result for system 2 stems not only from a flawed geometry of the outer ring but also from the undesirable action of a component or components of the system.

In the case of measurements of bearing 6205ZZ, which generated a relatively greater level of vibration, it is difficult to indicate a correlation for results for system 1 and 2 . Still, results for system 1 are more similar to those for the reference system in both cases. Note that system 2 sometimes has excessively high results and too low results other times depending on the degree of damage in the bearing. Results for system 1 'interwove' with correct values.

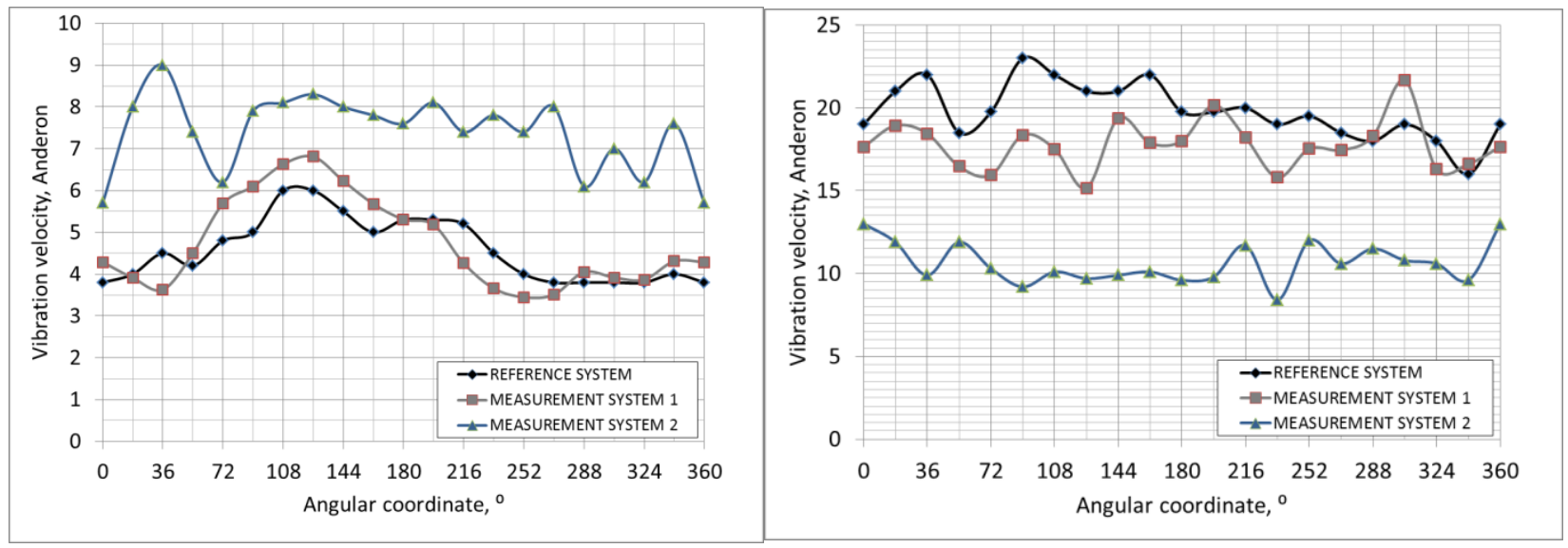

Fig. 4. Results of measurements of the RMS value for points on the perimeter of the outer ring. Medium band: bearing $6204 \mathrm{ZZ}$ on the left-hand side and bearing $6205 \mathrm{ZZ}$ on the right-hand side

A metrological analysis was performed (Tab. 1) for a more accurate comparison of the results. An arithmetic mean and dispersion of the results as mean square error and range were calculated to assess the overall difference in the results. The Pearson correlation coefficient determined the nature of the dependence between the results.

Additionally, the relative error for results at each point for the tested systems was calculated in relation to the reference system:

$$
w_{\Delta z}=\frac{\Delta Z_{p}-\Delta Z_{a}}{\Delta Z_{a}}
$$

where:

$\Delta Z_{p}$ - the vibration level at a specific point on the outer ring measured by an investigated system, $\Delta Z_{a}$ - the vibration level at a specific point on the outer ring measured by the reference system.

The table includes also the arithmetic mean and the mean square error of the calculated relative errors. These calculations were used to determine a very useful Measurement Accuracy defined as the convergence of results with those of the reference instrument:

$$
M A=\left|\bar{w}_{\Delta z} \pm k_{p} s\right| \cdot 100 \%
$$

where:

$w_{\Delta z}$ - the arithmetic mean of relative errors of RMS value measurements at specific points of the outer ring of a bearing,

$k_{p}$ - coverage factor for the probability of 0.95 ,

$s$ - the square mean error of relative errors of RMS value measurements at specific points of the outer ring of a bearing. 


\begin{tabular}{|c|c|c|c|c|c|c|}
\hline Bearing & \multicolumn{3}{|c|}{$6204 Z Z$} & \multicolumn{3}{|c|}{$6205 Z Z$} \\
\hline Measurement system & tested 1 & tested 2 & reference & tested 1 & tested 2 & reference \\
\hline Arithmetic mean, And & 4.73 & 7.40 & 4.58 & 17.78 & 10.65 & 19.80 \\
\hline Mean square error, And & 1.08 & 0.91 & 0.76 & 1.47 & 1.21 & 1.63 \\
\hline Range, And & 3.36 & 3.30 & 2.20 & 6.51 & 4.60 & 7.00 \\
\hline Pearson correlation coefficient & 0.85 & 0.54 & & 0.20 & -0.25 & \\
\hline Arithmetic mean of relative errors & -0.03 & -0.64 & & 0.10 & 0.46 & \\
\hline Mean square error of relative errors & 0.12 & 0.24 & & 0.10 & 0.09 & \\
\hline System measurement accuracy, \% & 40.33 & 134.65 & & 38.67 & 71.24 & \\
\hline
\end{tabular}

Table 1. Results of the metrological analysis of the measurements

\section{Conclusions}

The results indicate that the accuracy of some systems may be susceptible to the degree of damage in a bearing. The comparison of the arithmetic means clearly indicates that the tested system 1 offers results that are much closer to those of the reference system. The analysis of dispersion did not yield clear-cut results. The reference system has a lower dispersion of results for the operational bearing but larger for the defective one. This is because bearing 6204ZZ did not have significant defects in its outer ring that could result in a difference in measurement results for various points. The range for the reference system should result only from deformations in outer race geometry and so it should be minimal. Larger ranges in results from the perimeter may result from the incorrect operation of the measurement system.

As was mentioned above, bearing $6205 \mathrm{ZZ}$ generated an excessive vibration level caused by the inaccurate geometry of the race in the outer ring. The small range for system 2 indicates an incorrect operation of the device as it failed to detect a change in the vibration level near the abnormal geometry location on the surface of the race. In the case of the better-quality bearing, system 1 correlates strongly with the reference system, while system 2, only moderately. In the case of the defective bearing, results from both systems fail to interrelate with the reference system and the correlation is weak. Moreover, the correlation for system 2 becomes negative.

Eventually, the measurement accuracy for system 1 is the same for both bearings and amounts to $40 \%$. The susceptibility of the system to the measurement of diversified vibration level is minor. The accuracy of system 2 is over three times larger for measurements of a non-defective bearing. The result is much better for the bearing that generated excessive vibration, which is still worse than for system 1 by 33 percentage points. The high susceptibility of the system to the level of bearing vibration may result from the fact that the whole measurement chain (including sensor mounting) are worse suited to measure lower vibration velocities.

The presented method employed basic statistical tools and was intended as an illustration. It may be used to compare measurement systems quickly. To perform a more in-depth analysis in the future, additional system characteristics could be taken into consideration such as repeatability, reproducibility, or measurement hysteresis or other vibration assessment parameters such as the peak value or SPM. Further studies should involve a greater number of bearings, determination of the degree of damage, and tests of a greater number of types and sizes of rolling bearings. The most important aspect of the study should, however, be the determination (based on available sources and experience) of the vibration limits acceptable during quality control.

Studies involving the comparison of measurement systems could be of particular interest to purchasers of bearings who may want to verify the performance parameters of the products for various reasons such as a change of the supplier. The simple example of multiple measurements of a single bearing shows that despite constant monitoring of equipment, differences in results occur. The differences in the designs of the stations are too significant to suggest any design solutions at this stage. The awareness of the differences in measurement results will be a valuable factor affecting the selection of the best design solutions that together will form a measurement station that will detect rolling bearing vibration with high fidelity.

\section{Acknowledgement}

This publication is a result of joint research and development at FabrykaŁożyskTocznychKraśnik S.A. and the Kielce University of Technology under project 'Establishment of an R\&D Centre at FabrykaŁożyskTocznychKraśnik S.A.' under the Smart Growth Operational Programme 2014-2020 co-founded by the European Regional Development Fund No. CBR/1/50-52/2017 of 07.04.2017.

\section{Literature references}

[1] Morhain, A.;Mba, D.; (2003). Bearing defect diagnosis and acoustic emission; Proceedings of the Institution of Mechanical Engineers, Part J: Journal of Engineering Tribology Vol 217, Issue 4, pp. 257 - 272. 
[2] Tong,Q.; Cao,J.;Han; B.;Wang, D.;Lin, Y.; Zhang, W.; Wang J.; (2017). A fault diagnosis approach for rolling element bearings based ondual-tree complex wavelet packettransform-improved intrinsictime-scale decomposition, singularvalue decomposition, and onlinesequential extreme learning machine; Advances in Mechanical Engineering2017, Vol. 9(12), pp. 1-12.

[3] Serrano Mira, J.; BruscasBellido, G. M.; AbellanNebot, J. V. \& Romero Subiron, F.; (2010). Influence of geometric defects in bearing outer race on vibration generation: an oriented study for manufacturing tolerances specification and allocation; Annals of DAAAM for 2010 \& Proceedings of the 21st International DAAAM Symposium, Volume 21, No. 1.

[4] Kankar, P.K.; Satish, C. Sharma; HarshaS.P.; (2011). Fault diagnosis of ball bearings using continuous wavelet transform; Applied Soft ComputingVolume 11, Issue 2, March 2011, pp. 2300-2312.

[5] Nassif, H.H.;Gindy, M.; Davis J.; (2005).Comparison of laser Doppler vibrometer with contact sensors for monitoring bridge deflection and vibration; NDT \& E International Volume 38, Issue 3, pp. 213-218.

[6] Rodriguez, R.M.; Cristalli, C.; Paone, N.; (2002). Comparative study between laser vibrometer and accelerometer measurements for mechanical fault detection of electric motors; Proc. SPIE 4827, Fifth International Conference on Vibration Measurements by Laser Techniques: Advances and Applications, 10.1117/12.468154.

[7] Aye, S.A.; (2012). Bearing damage characterization using SVAN 958 and laser in the time domain; 10th intl Conference on Vibration Measurements by Laser and Noncontact Techniques, pp. 398-404.

[8] Tallian, T.E.; Gustafsson, O.G.; (2008). Progress in Rolling Bearing Vibration Research and Control. A S L E Transactions 8:3, pp. 195-207.

[9] Adamczak, S.; Zmarzły, P.; (2017). Influence of Racewa waviness on the level of vibration in rolling-element bearings; Bulletin of the Polish Academy of Sciences Technical Sciences Volume 64, Issue 4, pp. 541-551.

[10] SUGAWARA Laboratories Inc.; Bearing inspectionsystemsAnderonmeter, wavimeter, roundness measurement device, bearing life testing machine, pusher-type torque sensor, and calibrator(documentation of SUGAWARA company).

[11] Adamczak, S.; Zmarzły, P.; Stepień, K.; (2016). Identification and analysis of optimal method parameters of the V-block waviness measurements. Bulletin of the Polish Academy of Sciences Technical Sciences, 64, pp.325-332.

[12] Lacey, S.J.; (2008). An overview of Bearing Vibration Analysis;Maintnance\& Asset Man-agement vol. 23 no 6 pp. 32-42.

[13] Standard ISO 15242-1:2015, "Rolling bearings - Measuring methods for vibration - Part 1: Fundamentals".

[14] Standard ISO 15242-2:2015, "Rolling bearings - Measuring methods for vibration - Part 2: Radial ball bearings with cylindrical bore and outside surface".

[15] Noise and vibration tester MVH 90E, MVH 200E MVH 90EL, MVH 200EL (documentation of SKF company)

[16] Producers website: https://chinazys.en.made-in-china.com/product/BSYElReyCzcD/China-Zys-High-PerformanceBearing-Vibration-Measuring-Instrument.html (01.08.2018).

[17] Producers website: https://chinazys.en.made-in-china.com/product/BSYElReyCzcD/China-Zys-HighPerformance-Bearing-Vibration-Measuring-Instrument.html (01.08.2018).

[18] Producers website: http://www.baltech.biz/catalog.php?catalog=19 (01.08.2018).

[19] Adamczak, S.; Wrzochal, M.; (2018). Comparative study of selected bearing vibration measuring systems; Mechanik 7/2018, pp. 498-500.

[20] Inoue, H.;(2014). Anderon vibration” SUGAWARA Laboratories Inc. 2014;(documentation of SUGAWARA company). 